\title{
The optimization design of Hall compo- nent array based on genetic algorithm
}

\author{
Weiwei Zhai ${ }^{1,2}$, Wenzhu Gao ${ }^{1}$, Yu Zhang ${ }_{\text {Zhang }^{1 *}}{ }^{* *}, \mathrm{Ke} \mathrm{Bi}^{1}$, Tianyue $\mathrm{Xu}^{1}$, Tieqiang \\ ${ }^{1}$ College of physics, JiLin University, Changchun, China \\ ${ }^{2}$ College of electronic science \& engineering, JiLin University, Changchun, China
}

\begin{abstract}
It can greatly shorten the design period and make optimization more reasonable that use genetic algorithm for the Hall component array optimization design. The method is to set up the optimized platform based on ANSYS and MATLAB software. MATLAB software is used to realize the program design of genetic algorithm, ANSYS software is used to realize the simulation of hall component and get the corresponding hall voltage. With calling back the hall voltage of ANSYS, MATLAB can obtain the best design parameters after gradually choice. To avoid premature phenomenon of genetic algorithm, this paper also take sequencing selection method, novel crossover and mutation operation.
\end{abstract}

Keywords: Genetic algorithm, Hall component, Optimization design

\section{Introduction}

The Hall component is a magnetic susceptibility component. It is widely used in the electromagnetic measurement, the power measurement, automatic control and communications devices [1]. Hall voltage will be affected by the structural dimensions of Hall component. Such as the length to width ratio, the thickness, the length and the width of the electrode of Hall component. At present, the impact of a single factor is proposed, but there is little study on optimal combination of all influence factors, so empirical way are used for hall component designs, which make it difficult to obtain the best parameter. Genetic algorithm is a kind of simulation natural evolution bionic algorithm. It incentive good structures by the principle of Darwin's survival of the fittest. In the iteration process, it keep the existing structure and looking for better with the simulation of Mendel's genetic variation theory [2]. In a word, the genetic algorithm transform multi-factor problem into $\mathrm{N}$ dimensional variable problem and obtain the best combination at last. This algorithm also has fast convergent rate and accurate computing speed.

For the traditional genetic algorithm often leads to immature convergence, this essay improves a real-coded genetic algorithm. The algorithm takes the selection sort method, novel crossover and mutation operation, which avoid the premature phenomenon effectively.

\section{Optimization design principle}

For nonlinear function $f(\mathrm{X})$, the global optimization problem is:

$\operatorname{Min}(\max ) f(\mathrm{x})$

$$
\text { Where:, } a_{i}<x_{i}<b_{i}, a_{i} \text { and } b_{i} \text { are the }
$$

upper and lower limits of the variable $x_{i}$. The method that uses genetic algorithms 
to accomplish the optimal parameter values solution is as follow.

Input the initial parameter values firstly, and then produce a number of chromosomes after initialization. Thirdly, aftersorting and selecting on chromosome, the crossover and mutation operations should be employed. Repeat this process until meet the satisfied solution [3].

\subsection{Coding mode}

While the unknown variables are discrete, use the binary coding. For the continuous variable, solving accuracy of binary encoding is affected by the limit of chromosome length, therefore, we select the decimal coding mode and the variable constitute a chromosome in turn:

$V=x_{1}, x_{2}, \ldots x_{n}$

\section{2 selection}

In the selection, sort the chromosome sequence according to the serial number. The smaller serial number is, the more optimal chromosome is. Define the Selected probability $p_{i}$ of chromosome $V_{i}$ whose serial number is $i$ as follow:

$p_{i}=\frac{1}{\text { size }}+\alpha($ gen $) \frac{\text { size }+1-2 i}{\text { size }(\text { size }+1)}$

Where, size is population size, $\alpha(g e n)$ is function of generations gen, which is determined by the difference probability between adjacent two chromosomes in populations.

$$
\alpha(\text { gen })=\left\{\begin{array}{c}
0.2 \quad 0 \leq \text { gen }<a \\
0.4 \quad a \leq \text { gen }<b \\
0.6 \quad \text { gen } \geq b
\end{array}\right.
$$

Here, $a, b$ are appropriate generations. In the early evolution, for avoiding premature phenomenon and keeping the diversity of the population, $\alpha($ gen $)$ select small value. In the middle of the evolution, for competitiveness is reduced between adjacent two chromosomes, so $\alpha$ (gen) should has bigger value to prevent random search.

\subsection{Intersect}

Generate a random number $r$ between [0,1]. If $r<P_{c}, P_{c}$ is crossover probability, choose $V_{i}$ as father generation. Put the father generation chromosome in pairing randomly. For example, there are two father generation $\left(V_{1}, V_{3}\right)$, their offsprings $V_{1}^{\prime}$ and $V_{3}^{\prime}$ are as follow:

$\left\{\begin{array}{l}V_{1}^{\prime}=c V_{1}+(1-c) V_{3} \\ V_{3}^{\prime}=c V_{3}+(1-c) V_{1}\end{array}\right.$

Where, $c$ is a random number between $[0,1]$. It should be noted, if the chromosomes involved in the intersect are very similar or the same, it is not generate new mode. So to define the distance between the two chromosomes is:

$$
d=\sqrt{\sum_{i=1}^{n}\left(x_{i}^{(1)}-x_{i}^{(3)}\right)^{2}}
$$

Where, $x_{i}^{(1)} 、 x_{i}^{(3)}$ are variable of $V_{1}$ and $V_{2}$.

\subsection{Variation}

Variation is the operation that choose variable chromosome according to the mutation probability $P_{m}$ in the cross population. Generate a random number $R$ between range 0 to 1 , if $R<P_{m}$, the chromosome is selected as a variation chromosome. For the positive integer $M$ and Random variation direction $k$, the 
offspring $V_{i}^{\prime \prime}=V_{i}^{\prime}+M \cdot k$. Check whether it is feasibly. If so, $V_{i}^{\prime}=V_{i}^{\prime \prime}$, otherwise variation again [4].

\section{The optimization design of Hall component array}

\subsection{Optimization design platform}

Hall component design platform based on genetic algorithms is composed of MATLAB and ANSYS software together.

In this essay, use ANSYS to simulate the performance of Hall component under different sizes. ANSYS software can simulate the Hall voltage generated by different conditions, which can more intuitively and more accurately analysis impacts of the Hall component. MATLAB has great advantage in implementing the complex algorithms design. Use MATLAB software to design the program of genetic algorithms, meanwhile call ANSYS to simulate the corresponding Hall component array and obtain the corresponding Hall voltage. (a)

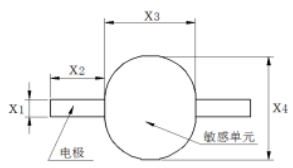

(c)

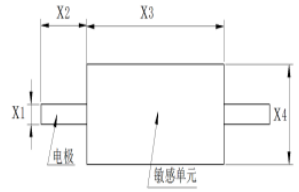

(b)

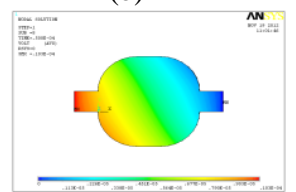

(d)

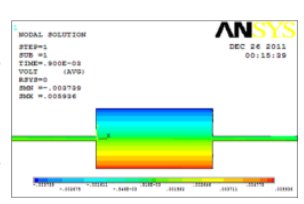

Fig. 1: (a) the schematic diagram of rectangular Hall component (b) ANSYS simulation results diagram of rectangular Hall component (c) the schematic diagram of elliptic Hall component (d) the ANSYS simulation results diagram of elliptic Hall component.

\subsection{Selection of genetic parameters}

According to the classical formula of the Hall voltage [5]:

$$
U_{H}=R_{H} \frac{I B}{b}
$$

Where, $\quad R_{H}=1 /(n q) \quad$ is Hall coefficient. $I$ is the control current that ventilation in Hall component, $B$ is magnetic field intensity which perpendicular to the direction of control current, $b$ is the thickness of the Hall component. From the formula 7 , we know that the materials of components, the thickness, and the current accessed to and the strength of magnetic field have effected on the size of the Hall voltage. But the formula 7 only apply to the general situation and the approximate calculation, a more useful expression of Hall voltage which needs to introduce a geometric correction factor $G$ in the formula 7 , namely :

$U_{H}=G R_{H} \frac{I B}{b}$

Geometric correction factor $G$ will be different according to Hall component shape, the Hall voltage value calculated by ANSYS software.

Select multiple parameters which need to be optimized as input variables. Population size chooses 500, the maximum iterations number is 2000 . Crossover probability $P_{c}$ and mutation probability $P_{m}$ changed with the evolution generations:

$\left\{\begin{array}{l}P_{c}^{(g e n)}=P_{c}^{(g e n-1)}-\left(P_{c}^{(0)}-0.2\right) / \max g e n \\ P_{m}^{(g e n)}=P_{m}^{(g e n-1)}-\left(0.5-P_{m}^{(0)}\right) / \max g e n\end{array}\right.$

Where, $P_{c}^{(0)}=0.8, \quad P_{m}^{(0)}=0.1$, max gen is the maximum evolution 
generations, variation parameter $M$ :

$$
M=\frac{f_{i}}{\operatorname{avgf}}\left(1-\frac{\operatorname{gen}}{\max g e n}\right)
$$

Where, $f_{i}$ is the objective function value of variation chromosome. Here the electromagnetic calculation results by the ANSYS software. $a v g f$ is the mean value of all chromosomes objective function.

\section{Analysis of Results}

In order to verify the feasibility of the proposed method, optimize for the rectangular structure of the Hall element array. Select a single Hall element of length is $1 \mathrm{~mm}$. Optimization seen that the best single Hall element width is $0.625 \mathrm{~mm}$, electrode width is $0.1 \mathrm{~mm}$, the maximum electrode length is $5 \mathrm{~mm}$. Through single factor analysis, we known that the results were better when its length and width ratio between 1.5 and 2 . Due to short circuit, when the aspect ratio value of the Hall device is too small, it will have a great impact on the Hall voltage. For the aspect ratio is greater than 2.0, the resistance between control current of electrodes and input power dissipation are increased. Thereby, it will reduce the output voltage of the Hall component and affect the performance. Within a certain range, the Hall voltage will increase as the electrode of the Hall component length increased. However, due to the sizes of the electrode have a significant impact on the geometric correction factor $G$, the increase of electrode should not be too long.
However, if the width of the electrode takes less than one-tenth the size of element length, it also can avoid the impact of short-circuit. Thus, it is verified the effectiveness of this method.

In a word, the optimized design method based on genetic algorithms, not only greatly simplifies the design cycle, but also make optimization more convenient, accurate and reasonable.

\section{References}

(1) Xu Shengrui, Zhou Xiaowei, etc, "Particular electrical quality of aplane $\mathrm{GaN}$ films grown on r-plane sapphire by metal-organic chemical vapor deposition", Journal of Semiconductors, 30(11), 2009.

(2) Ma Xiaoshu, Li Yulong, Yan Lang, "Comparsion review of traditional multi-objective optimization methods and multi-objective genetic algorithm", Electric drive automation, 32(3), pp:48-50, 2010.

(3) Yan Lewei, Chen Shuhui, "Solving Nonlinear Equations Based on Improved Genetic Algorithm", Acta Scientiarum Naturalium Universitatis Sunyatsent, 50(1), 2011.

(4) Song Mingxin, Gong Chunqing, etc "Study on the Effects of Shape Parameters on MEMS Hall Device Properties", Chinese Journal of Sensors and Actuators, No. 5, 2006.

(5) M. Yamagishi, J. Soeda, T. Uemura, etc., "Free-electron-like Hall effect in high-mobility organic thin-film transistors", Physical review B 81 , 161306(R), 2010. 